\title{
Verbraucherforschung wird wichtiger
}

\author{
Christian Grugel
}

Online publiziert: 5 Mai 2012

(C) Bundesamt für Verbraucherschutz und Lebensmittelsicherheit (BVL) 2012

Die Verhältnisse in Wirtschaft und Gesellschaft verändern sich seit Beginn der neunziger Jahre mit atemberaubender Geschwindigkeit. Ursachen hierfür sind Megatrends wie die Entstehung der Informationsgesellschaft, die Globalisierung, die Entwicklung von Demographie und Migration, die unsichere Verfügbarkeit kritischer Ressourcen wie Energie, bestimmte Rohstoffe und Agrarprodukte, der Klimawandel und der zunehmende Einfluss der Finanz- auf die Realwirtschaft. Im Zuge dieser Veränderungen hat die Verbraucherpolitik einen erkennbaren Bedeutungszuwachs erfahren. Dieser ist darauf zurückzuführen, dass Sicherheit und Selbstbestimmung zentrale Ziele einer Verbraucherpolitik geworden sind, die Markwirtschaft als Wirtschaftspolitik aus der Perspektive der Verbraucher versteht. Diese Einschätzung stützt sich auch auf die Erkenntnis, dass die Wirtschaft nur bis zu der Größe wachsen kann, die am Ende der Wertschöpfungskette durch die Summe der angebotenen Waren, Dienstleistungen und Rechte bestimmt wird, die von Verbrauchern tatsächlich gekauft werden.

Die aktuelle Situation der Verbraucherpolitik kann mit der Lage der Umweltpolitik zum Ende der achtziger Jahre verglichen werden. Nach dem Unfall im sowjetischen Kernreaktor Tschernobyl legten Bund und Länder die Zuständigkeiten für Kernenergie, Wasser- und Abfallwirtschaft, Luftreinhaltung,

Dr. Ch. Grugel ( $\square)$

Bundesministerium für Ernährung, Landwirtschaft und Verbraucherschutz (BMELV), Abteilung

Verbraucherpolitik, Wilhelmstraße 54,

10117 Berlin, Germany

e-mail: christian.grugel@bmelv.bund.de
Chemikalienrecht sowie Naturschutz in Ministerien zusammen, die den Umweltschutz in ihrer Bezeichnung führten. Mit der späteren Umbenennung der „Ministerien für Umweltschutz“ in „Umweltministerien“ wandelte sich die eher reaktive, auf die Bewahrung der Umwelt gerichtete Politik zu einer gestaltenden Umweltpolitik. Parallel dazu entstanden die Umweltwissenschaften, in denen konvergierende Fragestellungen zur Umwelt aus den Natur-, Ingenieurund Rechtswissenschaften sowie der Medizin bearbeitet wurden.

Das Auftreten von BSE in Europa hat zu Beginn des letzten Jahrzehnts zu einer mit dem Umweltschutz vergleichbaren Bedeutung des Verbraucherschutzes geführt. In Analogie zur Entwicklung in der Umweltpolitik wurden auch die Aufgaben des Verbraucherschutzes jetzt überwiegend in „Ministerien für Verbraucherschutz “ zusammengeführt und die Fachverwaltungen reorganisiert. Lag der Schwerpunkt zunächst beim gesundheitlichen Verbraucherschutz ist nach dem Zusammenbruch der Investmentbank Lehman Brothers und der dadurch ausgelösten Finanzkrise sowie immer neuen Problemen beim Datenschutz im Internet jetzt auch der wirtschaftliche Verbraucherschutz stärker in den Mittelpunkt der öffentlichen Wahrnehmung gerückt.

Anders als die Umweltwissenschaften werden Verbraucherwissenschaften bisher nur von einer Minderheit als eigenständiger, interdisziplinärer Wissenschaftszweig wahrgenommen, auch wenn sich die Rechtswissenschaften, die Wirtschaftswissenschaften und nicht zuletzt die Gesellschaftsund Sozialwissenschaften seit langem auch mit Fragen des Verbraucherschutzes beschäftigen. Verbraucherwissenschaften sind, ähnlich wie die 
Umweltwissenschaften auf eine breite interdisziplinäre Zusammenarbeit angewiesen. Erkenntnisgewinne sind vor allem dort zu erwarten, wo wissenschaftliche Fragestellungen in der Zusammenarbeit aller betroffenen Disziplinen bearbeitet werden.

Neben ruhigen Phasen kennt die Wissenschaft auch Entwicklungen, die grundlegend neue Erkenntnisse oder Methoden liefern. Aktuell erleben wir dies im Bereich der Verbraucherwissenschaften. Bereits seit Jahrzehnten greifen die Wirtschafts- und Sozialwissenschaften auf Selbstorganisationsmodelle zurück, die zuerst in den Naturwissenschaften entwickelt wurden und die zu einer Zusammenführung der auseinanderstrebenden Disziplinen der Wissenschaft geführt haben. Es sind die gleichen Modelle, die auf die Strukturbildung bei der neuronalen Vernetzung des menschlichen Gehirns, die Vernetzung von Internetseiten oder die Beschreibung des kollektiven Verhaltens in Schwärmen angewandt werden können. Diese Methoden sind geeignet, auch turbulente Entwicklungen in komplexen Systemen zu beschreiben und die Grenzen der Vorhersagbarkeit von Prozessen aufzuzeigen. Auch die Möglichkeit, Hirnaktivitäten zu beobachten und zu interpretieren hat das Methodenspektrum der Verbraucherforschung erweitert.

In den Rechtswissenschaften werden diese Methoden bisher kaum angewandt. Grundlegende Fragen der Rechtswissenschaften werden durch analytische Verfahren, zum Beispiel unter Einsatz der Rechtsvergleichung bearbeitet und die Rechtsanwendung zumeist über die Analyse von Gerichtsurteilen untersucht. Studien, die sich prospektiv mit den Auswirkungen einer bestimmten Rechtssetzung beschäftigen und dabei auch berücksichtigen, welchen Organisationsaufwand die Regelungen verursachen, in welchem Umfang sie voraussichtlich eingehalten werden, ob Umgehungsmöglichkeiten bestehen und welche Folgen sich daraus ergeben, werden seltener durchgeführt. Gerade solche Studien können bei interdisziplinärer Zusammenarbeit in den Verbraucherwissenschaften aber wertvolle Erkenntnisse für die zweckmäßige Ausgestaltung von Rechtsnormen liefern.

Es ist naheliegend, dass sich auch das Bundesministerium für Ernährung, Landwirtschaft und Verbraucherschutz mit der Frage beschäftigt hat, welche Möglichkeiten für eine wissenschaftliche Absicherung des Regierungshandels durch die Verbraucherwissenschaften bestehen. Bei diesen Überlegungen steht das Bundesministerium nicht allein. Auch der Bundestag, einzelne Landesministerien und politische Parteien mit ihren Stiftungen beschäftigen sich zunehmend mit der Frage, welchen Beitrag die Verbraucherwissenschaften durch Modellbildung und evidenzbasierte Untersuchungen leisten können.

Seit knapp zehn Jahren wird das Bundesministerium durch den Wissenschaftlichen Beirat für Verbraucherschutz und Ernährung beraten. Zum Januar 2012 wurden die Mitglieder des Beirats unter Berücksichtigung der aktuellen verbraucherpolitischen Herausforderungen neu berufen. Dabei wurde die Expertise im Bereich der Verhaltensökonomik vergrößert. Der Beirat berät das Bundesministerium insbesondere in strategischen Fragen.

Während dem Bundesministerium in der Agrarpolitik die Ressortforschung zur Politikberatung zur Verfügung steht, fehlt in der Verbraucherpolitik ein entsprechendes Instrumentarium. Aktueller Entscheidungshilfebedarf wird deshalb über Gutachten abgedeckt, die einzeln ausgeschrieben werden. Im Vergleich zur europäischen oder internationalen verbraucherpolitisch ausgerichteten Forschung sind die Verbraucherwissenschaften in der deutschen Forschungslandschaft weniger ausgeprägt und auf unterschiedliche Standorte verteilt. Um diesen Nachteilen zu begegnen, hat das Bundesministerium für die in der Verbraucherforschung aktiven Wissenschaftler und Forschergruppen das „Netzwerk Verbraucherforschung“ als Plattform für den interdisziplinären, verbraucherwissenschaftlichen Austausch und als Anlaufstelle für interdisziplinäre Themen initiiert. Das Netzwerk soll zum Kristallisationspunkt für die Verbraucherforschung werden. Es wird durch eine beim Bundesamt für Verbraucherschutz und Lebensmittelsicherheit eingerichtete Geschäftsstelle unterstützt. Ihre Aufgaben für das Netzwerk bestehen insbesondere darin, die Zusammenarbeit der Wissenschaftler und Forschergruppen zu unterstützen, Fachforen zu organisieren und zur Themenfindung beizutragen. Wissenschaftler aus dem Netzwerk können ihr Interesse an der Aufnahme in einen Expertenpool bekunden, damit beschränkte Ausschreibungen für Entscheidungshilfebedarf des Bundesministeriums schneller adressiert werden können.

Als weitere Maßnahme zur besseren Abdeckung des eigenen Entscheidungshilfebedarfs bei verbraucherrechtlichen Fragen, hatte das Bundesministerium ein Projekt ausgeschrieben, das von der Universität Bayreuth gewonnen wurde. Über das Projekt wird für mehrere Jahre eine Stiftungsprofessur gefördert, an der Entscheidungshilfevorhaben des Bundesministeriums bearbeitet werden. Die Universität Bayreuth 
hat sich verpflichtet, die Ausrichtung der Forschung auf diesem Lehrstuhl auch nach Auslaufen der Förderung beizubehalten. Bundesministerium und Universität profitieren davon, dass wegen der Schwerpunktsetzung ein Kompetenzzuwachs in verbraucherrechtlichen Fragen entsteht, der anders kaum zu erreichen ist. Die ersten Ergebnisse sind ermutigend. Es wird zu prüfen sein, ob diese Verfahrensweise auch auf andere Forschungsgebiete der Verbraucherwissenschaften übertragen werden kann. 